\title{
ARC TAN- EXPONENTIAL TYPE DISTRIBUTION INDUCED BY STEREOGRAPHIC PROJECTION / BILINEAR TRANSFORMATION ON MODIFIED WRAPPED EXPONENTIAL DISTRIBUTION
}

\author{
Y. PHANI, S.V.S.GIRIJA AND A.V. DATTATREYA RAO
}

\begin{abstract}
In this paper we make an attempt to construct a new three parameter linear model, we call this new model as Arc Tan-Exponential Type distribution, by applying Stereographic Projection or equivalently Bilinear transformation on Wrapped Exponential distribution, Probability density and cumulative distribution functions of this new model are presented and their graphs are plotted for various values of parameters.
\end{abstract}

Mathematics Subject Classification 2000: 60E05, 62H11

Additional Key Words and Phrases: Cauchy Distribution, Circular models, Probability Distribution, Stereographic Projection, Wrapped Exponential Distribution.

\section{INTRODUCTION}

Minh and Farnum (2003) use Bilinear transformations /Stereographic projection to map points on the unit circle in the complex plane onto points $x$ on the real line Given any density function $g(\theta)$ on the interval $(-\pi, \pi)$ they show how a corresponding density function $f(x)$ on real line is induced. On these lines we make an attempt to construct a new linear model, Arc Tan-Exponential Type distribution, by slightly modifying the domain of the well Known Wrapped Exponential distribution [S.Rao Jammalamadaka (2001)].It is also observed that Cauchy's distribution is a special case of Arc Tan Exponential Type distribution.

\section{METHODOLOGY OF STEREOGRAPHIC PROJECTION/ BILINEAR TRANSFORMATION}

Stereographic Projection / Bilinear Transformation is defined by a one to one mapping given by $T(\theta)=x=u+v \tan \left(\frac{\theta}{2}\right)$, where, $\theta \in(-\pi, \pi), u, v \in$, and $v>0$ .Suppose $\theta$ is randomly chosen on the interval $(-\pi, \pi)$. Let $G(\theta)$ and $g(\theta)$ denote the Cumulative distribution and probability density functions of the random variable $\theta$ respectively. Then $T(\theta)=x=u+v \tan \left(\frac{\theta}{2}\right)$, introduced by Minh and Farnum (2003) is a random point on the real line. Let $G(\theta)$ and $g(\theta)$ denote the Cumulative 
distribution and probability density functions of this random point $\theta$ respectively. Then $F(x)$ and $f(x)$ can be written in terms of $G(\theta)$ and $g(\theta)$ using the following Theorem.

Theorem 2.1: For $v>0$,

i) $F(x)=G(\theta(x))$;

ii) $\quad f(x)=g(\theta(x)) \frac{2}{v\left[1+\left(\frac{x-u}{v}\right)^{2}\right]}=g(\theta(x)) \frac{2 v}{\left(v^{2}+(x-u)^{2}\right)}$;

\section{WRAPPED EXPONENTIAL DISTRIBUTION}

A random variable $X_{W}$ on the unit circle is said to have wrapped exponential distribution with parameter $\lambda>0$, denoted by WE $(\lambda)$, if the probability density, cumulative distribution and characteristic functions are given respectively by

i) $g_{w}(\theta)=\frac{\lambda e^{-\lambda \theta}}{1-e^{-2 \pi \lambda}}, 0 \leq \theta<2 \pi$,

ii) $G_{w}(\theta)=\frac{1-e^{-\lambda \theta}}{1-e^{-2 \pi \lambda}}, 0 \leq \theta<2 \pi$, and

iii) $\phi_{p}=\frac{1}{1-(i p / \lambda)}, p=0, \pm 1, \pm 2, \pm 3, \ldots$

By modifying the domain of wrapped Exponential to $-\pi \leq \theta<\pi$, the modified version of the probability density and cumulative distribution functions are given by

1) $g(\theta)=\frac{\lambda \exp (-\lambda(\theta+\pi))}{(1-\exp (-2 \pi \lambda))}, \lambda>0$ and $-\pi \leq \theta<\pi$

2) $G(\theta)=\frac{1-\exp (-\lambda(\theta+\pi))}{1-\exp (-2 \pi \lambda)}, \quad \lambda>0$ and $-\pi \leq \theta<\pi$ 


\section{GENERATING THE ARC TAN-EXPONENTIAL TYPE DISTRIBUTION WHEN $\theta$ FOLLOWS MODIFIED WRAPPED EXPONENTIAL DISTRIBUTION}

Theorem 4.1: If $\theta$ follows Wrapped Exponential distribution in $(-\pi, \pi)$, then $x=T(\theta)=u+v \tan \left(\frac{\theta}{2}\right)$, has a 3 parameter Arc Tan-Exponential Type distribution.

Proof: As $\theta$ is Wrapped Exponentially distributed, $g(\theta)=\frac{\lambda \exp (-\lambda(\theta+\pi))}{(1-\exp (-2 \pi \lambda))}$, for all $\theta$ in $[-\pi, \pi)$.

Therefore, Theorem 2.1 yields,

$$
f(x)=\frac{v \lambda \operatorname{cosech}(\pi \lambda) \exp \left[-2 \lambda \tan ^{-1}\left(\frac{x-u}{v}\right)\right]}{\left(v^{2}+(x-u)^{2}\right)},-\infty<x<\infty
$$

This is the density function of the 3-parameter Arc Tan-Exponential Type distribution.

1. When $u=0$, we have 2-parameter Arc Tan-Exponential Type distribution

$$
f(x)=\frac{v \lambda \operatorname{cosech}(\pi \lambda) \exp \left[-2 \lambda \tan ^{-1}\left(\frac{x}{v}\right)\right]}{\left(v^{2}+x^{2}\right)},-\infty<x<\infty
$$

2. When $u=0$ and $\lambda \rightarrow 0^{+}, f(x) \rightarrow \frac{v}{\pi\left(x^{2}+v^{2}\right)}$, which is a

Cauchy distribution.

3. When $u=0, v=1$ and $\lambda \rightarrow 0^{+}, f(x) \rightarrow \frac{1}{\pi\left(1+x^{2}\right)}$,

which is a Standard Cauchy distribution

\section{ARC TAN-EXPONENTIAL TYPE DISTRIBUTIONS}


Definition: Let $X$ be a continuous random variable and $u, v>0$ and $\lambda>0$ be real numbers. Then $X$ is said to have an Arc Tan-Exponential Type distribution with parameters $u, v$ and $\lambda$ if the probability density and cumulative distribution functions are given respectively by

$$
f_{X}(x ; u, v, \lambda)=\frac{v \lambda \operatorname{cosech}(\pi \lambda) \exp \left[-2 \lambda \tan ^{-1}\left(\frac{x-u}{v}\right)\right]}{\left(v^{2}+(x-u)^{2}\right)}
$$

and

$$
\begin{aligned}
& F_{X}(x ; v, \lambda)=\frac{\operatorname{cosech}(\pi \lambda)\left[\exp (\pi \lambda)-\exp \left(2 \lambda \tan ^{-1}\left(\frac{x-u}{v}\right)\right)\right]}{2} \\
&-\infty<x<\infty, \text { and } u, v>0, \lambda>0
\end{aligned}
$$

6. GRAPHS OF PROBABILITY DENSITY AND CUMULATIVE DISTRIBUTION FUNCTIONS FOR VARIOUS VALUES OF THE PARAMETERS.

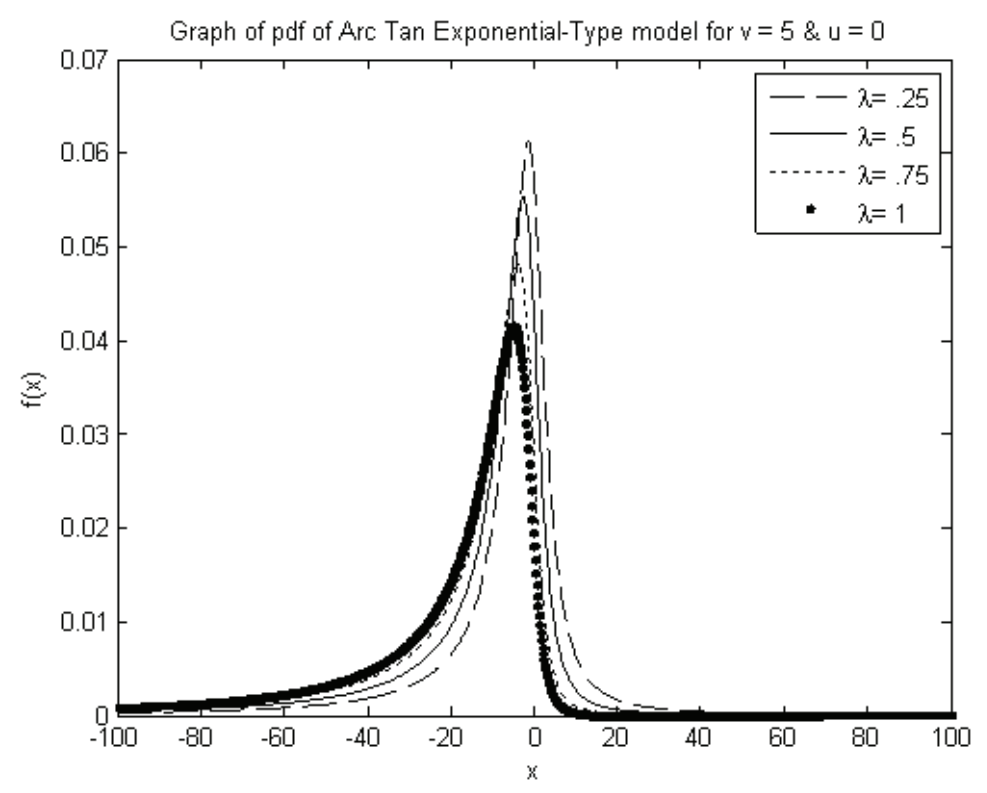

Fig-I 
ARC TAN-EXP. TYPE DISTRIB. INDUCED BY STEREOGR.

PROJECTION/BILINEAR TRANSF. ON MODIFIED WRAPPED EXPON. DISTRIB.

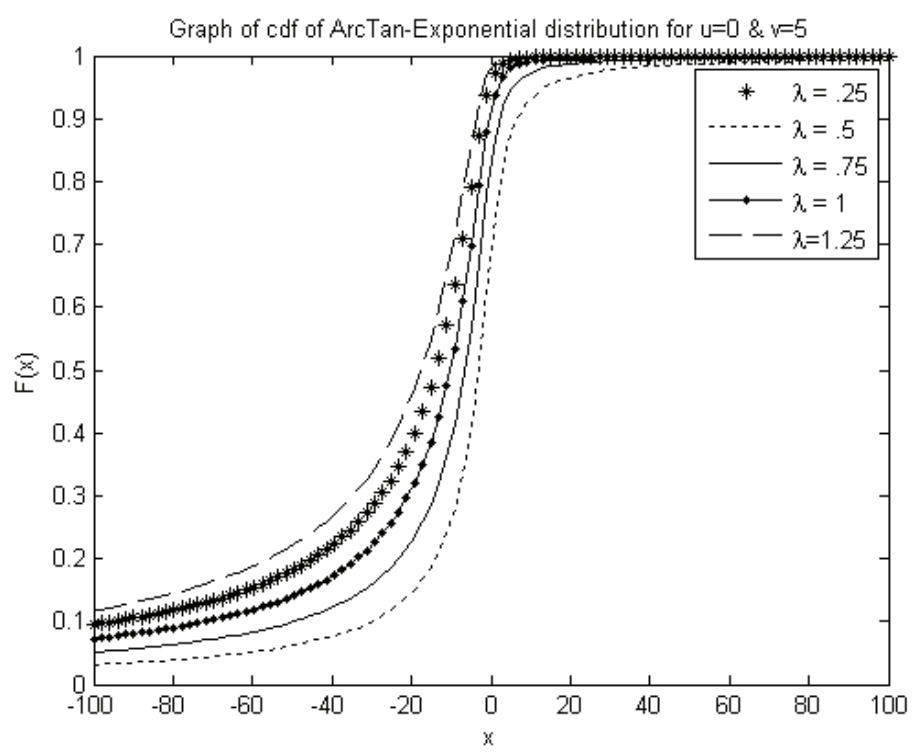

Fig-II

\section{ACKNOWLEDGMENT}

Authors would like to thank UGC, New Delhi, India for offering financial assistance to carry out the project under the head of Major Research Project no. F 41-785/2012 (SR) dt. 17-07-2012.

\section{REFERENCES}

1. Abramowitz, M.\& Stegun, I.A. (1965), Handbook of Mathematical Functions, Dover, New York.

2. Dattatreya Rao, A.V., Ramabhadra Sarma, I. and Girija, S.V.S. (2007), "On Wrapped Version of Some Life Testing Models", Comm Statist, - Theor.Meth. , 36, issue \# 11, pp.2027-2035.

3. Dattatreya Rao, A.V., Girija, S.V.S., Phani. Y. (2011), "Differential Approach to Cardioid Distribution", Computer Engineering and Intelligent Systems, Vol 2, No.8, pp. 1-6.

4. Girija, S.V.S., (2010), Construction of New Circular Models, VDM - VERLAG, Germany.

5. Jammalamadaka S. Rao and Sen Gupta, A. (2001), Topics in Circular Statistics, World Scientific Press, Singapore.

6. Mardia, K.V. and Jupp, P.E. (2000), Directional Statistics, John Wiley, Chichester. 


\section{K. GUPTA, V. AGRAWAL}

7. Minh, Do Le and Farnum, Nicholas R. (2003), "Using Bilinear Transformations to Induce Probability Distributions", Communication in Statistics - Theory and Methods, 32, 1, pp. 1 - 9.

8. Ramabhadrasarma, I. A.V.Dattatreya Rao and S.V.S.Girija (2009), "On Characteristic Functions of Wrapped Half Logistic and Binormal Distributions", International Journal of Statistics and Systems, Volume 4 Number 1, pp. 33-45.

9. Ramabhadrasarma, I. A.V.Dattatreya Rao and S.V.S.Girija (2011), On Characteristic Functions of Wrapped Lognormal and Weibull Distributions, Journal of Statistical Computation and Simulation, Vol. 81 , No. 5, 579-589.

10. S.Rao Jammalamadaka and Tomasz J. Kozubowski, (2001), “A Wrapped Exponential Circular Model”, Proc. Of AP Academy of Sciences, Vol.5, No.1,pp. 43-56.

Associate Professor of Mathematics,

Swarnandhra College of Engineering and Technology,

Narasapur, India.

Email: phaniyedlapalli23@gmail.com

Associate Professor of Mathematics, Hindu College,

Guntur, India.

Email: svs.girija@gmail.com

Professor of Statistics,

Acharya Nagarjuna University,

Guntur, India.

Email: avdrao@gmail.com

Received May 2013 